\title{
RESISTÊNCIA DE GENÓTIPOS DE BATATA A Phthorimaea operculella (Zeller)
}

\author{
Maria Teresa do Rêgo Lopes ${ }^{1 *}$; José Djair Vendramim² \\ ${ }^{1}$ Pós-Graduanda do Depto. de Entomologia, Fitopatologia e Zoologia Agrícola - USP/ESALQ. \\ ${ }^{2}$ Depto. de Entomologia, Fitopatologia e Zoologia Agrícola - USP/ESALQ, C.P. 9 - CEP: 13418-900 - Piracicaba, SP. \\ *Autor correspondente <edsonuep@secrel.com.br>
}

RESUMO: A traça Phthorimaea operculella (Zeller) é considerada atualmente uma das principais pragas da batata. O uso de resistência varietal para controle desse inseto é uma alternativa promissora, mas que ainda precisa ser melhor pesquisada. A resistência de genótipos de batata à traça foi avaliada, em condições de laboratório, analisando-se o efeito de folhas e tubérculos sobre a biologia do inseto. Comparou-se também o desenvolvimento da traça em folhas de batata e tomate. Os experimentos foram conduzidos com os clones de batata NYL 235-4 e N 140-201 (ambos com tricomas glandulares tipo A nas folhas), as cultivares Aracy, Apuã e Itararé e a cultivar de tomate Santa Clara. Os insetos foram criados em folíolos e tubérculos dos genótipos, avaliando-se a duração e a viabilidade das fases larval e pupal e o peso de pupas. $O$ desenvolvimento do inseto não foi afetado nas folhas dos genótipos de batata, mesmo nos materiais com tricomas glandulares. Entretanto, em folhas de tomate houve aumento do período larval e diminuição do peso de pupas, sendo este substrato menos favorável ao desenvolvimento da traça quando comparado às folhas de batata. Em testes com tubérculos, o clone NYL 235-4 apresentou resistência a $P$. operculella, provocando redução significativa na viabilidade larval e alongamento desta fase. O clone N 140-201 afetou o desenvolvimento do inseto de forma menos pronunciada, alongando a fase larval.

Palavras-chave: Solanum tuberosum, traça-da-batatinha, biologia, tricomas, tubérculos

\section{RESISTANCE OF POTATO GENOTYPES TO BY THE POTATO TUBER MOTH Phthorimaea operculella (Zeller)}

\begin{abstract}
The potato tuber moth, Phthorimaea operculella (Zeller) is today one of the most important pests in potato. The use of resistant varieties to control this insect is a interesting technique, but it is necessary more researches about it. Resistance of potato genotypes to this pest was investigated under laboratory conditions by evaluating the effect of leaves and tubers on insect biology. Potato and tomato leaves were also compared in relation to suitability of insect development. Tests were carried out with the following genotypes: clones NYL 235-4 and N 140-201 (both with type A glandular trichomes on leaves), cultivars Aracy, Apuã and Itararé. The tomato cultivar Santa Clara was also included. Insects were reared on leaflets and tubers of each genotype in order to evaluate larval and pupal development time, larval and pupal survival and pupal weight. Insect development was not affected by leaves of potato genotypes, even when glandular trichomes were present. However, tomato leaves increased larval development time and reduced pupal weight when compared to potato leaves. In tests with tubers, NYL 235-4 showed resistance to potato tuber moth with a reduction in larval survival and an increase in larval development time. N 140-201 tubers also increased larval development time when compared to the cultivars Aracy, Apuã and Itararé.
\end{abstract}

Key words: Solanum tuberosum, insect biology, trichomes, tubers

\section{INTRODUÇÃO}

A traça-da-batatinha Phthorimaea operculella é praga de várias solanáceas cultivadas incluindo batata, fumo, tomate, berinjela e pimentão (Varela \& Bernays, 1988). Em batata, as lagartas minam folhas e ramos e podem atacar tubérculos expostos no campo e durante o armazenamento, causando grande depreciação do produto (Radcliffe, 1982).

Devido ao emprego excessivo de inseticidas para controle dessa praga, principalmente na cultura da batata, várias pesquisas vêm sendo realizadas visando à obtenção de outros métodos de controle como a utilização de cultivares resistentes, que tem sido considerada uma alternativa promissora a ser incluída em programas de manejo integrado de pragas (Palacios et al., 1993).

A busca de fontes de resistência de batata a $P$. operculella tem sido realizada em vários países através da avaliação de espécies selvagens, cultivares primitivas e comerciais (Fenemore, 1980; Raman \& Palacios, 1982; Ojero \& Mueke, 1985; Gyawali, 1989; Ahmed et al., 1991; Gurr \& Symington, 1998; Stein, 1998).

Fatores de resistência encontrados em tubérculos de espécies selvagens têm sido transferidos com sucesso para germoplasmas cultivados (Chavez et al., 1988; Ortiz et al., 1990). Um outro mecanismo de defesa das plantas de batata são os tricomas glandulares presentes em folhas e ramos de espécies selvagens como Solanum berthaultii. Esta espécie apresenta dois 
tipos de tricomas, A e B, que liberam exsudatos que podem imobilizar pequenos artrópodes, impedir ou diminuir a alimentação, aumentar a mortalidade e afetar o desenvolvimento de algumas espécies de insetos (Tingey \& Gibson, 1978; Tingey \& Sinden, 1982; França \& Tingey, 1994).

Híbridos de $S$. berthaultii e $S$. tuberosum também têm demonstrado resistência a vários insetos, como é o caso do clone NYL 235-4, resistente a afídeos, cigarrinhas e aos besouros Leptinotarsa decemlineata e Epitrix spp. (Plaisted et al., 1992).

Em relação a $P$. operculella, entretanto, poucos trabalhos têm sido realizados para avaliar o efeito de genótipos com tricomas glandulares sobre o seu desenvolvimento (Musmeci et al., 1997; Westedt et al., 1998).

O objetivo do presente trabalho foi avaliar a resistência de genótipos de batata, incluindo alguns genótipos com tricomas glandulares, em relação a $P$. operculella.

\section{MATERIAL E MÉTODOS}

Foram avaliados os clones NYL 235-4 e N 140201 (oriundos de cruzamentos entre $S$. berthaultii e $S$. tuberosum), as cultivares Aracy, Apuã e Itararé de $S$. tuberosum e a cultivar Santa Clara de tomate. O clone NYL 235-4, desenvolvido pelo programa de melhoramento da Universidade de Cornell (Ithaca, NY, EUA), apresenta tricomas tipo $A$, com as mesmas características daqueles de $S$. berthaultii e tricomas tipo $B$ não funcionais, que não produzem gotas de exsudato (Plaisted et al., 1992). O clone N 140-201 também apresenta tricomas tipo A. As sementes destes genótipos foram fornecidas pela Embrapa Hortaliças, enquanto as das cultivares foram obtidas junto ao Instituto Agronômico de Campinas.

As folhas dos genótipos utilizadas no primeiro experimento foram provenientes de plantas cultivadas em casa de vegetação, em vasos plásticos com capacidade de $5 \mathrm{~L}$, enquanto os tubérculos foram oriundos de cultivo em condições de campo. A criação estoque de insetos foi mantida em condições de laboratório, em tubérculos de batata, cv. Bintje.

Inicialmente, foi comparada a biologia do inseto nos 5 genótipos de batata e na cultivar de tomate Santa Clara, utilizando-se folhas coletadas de plantas com 40 a 45 dias após o plantio. Folíolos de cada material, com o pecíolo envolvido por algodão umedecido para manter a turgidez, foram colocados individualmente em tubos de vidro $(8,5 \times 2,5 \mathrm{~cm})$. Foram colocadas três lagartas recém-eclodidas por tubo, num total de 60 lagartas por tratamento. Os tubos foram tamponados com algodão hidrófugo e os folíolos renovados diariamente a partir do $3^{\circ}$ dia. A ocorrência de pupas foi observada diariamente, sendo a pesagem feita após $24 \mathrm{~h}$. Foram avaliados a duração e viabilidade das fases larval e pupal e o peso de pupas machos e fêmeas.
Um segundo experimento foi instalado para avaliar a biologia da traça em tubérculos dos 5 genótipos de batata e em folhas da cultivar Apuã. Foram utilizados tubérculos (com cerca de 40 a $45 \mathrm{~g}$ ) previamente furados com alfinete para facilitar a penetração das lagartas. Cinco tubérculos de cada genótipo foram infestados com 20 lagartas recém-eclodidas, seguindo a proporção de uma lagarta para cada $2 \mathrm{~g}$ de batata, de acordo com as recomendações de Pratissoli et al. (1993). Os tubérculos infestados foram individualizados em recipientes de plástico $(0,6 \mathrm{~L})$, cobertos com voile fixo por elástico. No fundo dos recipientes, foi colocada uma camada de algodão como substrato para pupação. Uma semana após a infestação, os recipientes passaram a ser observados diariamente, registrando-se a ocorrência de pupas, que foram pesadas $24 \mathrm{~h}$ após a formação. No tratamento com folhas de 'Apuã', seguiu-se a mesma metodologia utilizada no primeiro experimento, mantendo-se 25 tubos com 4 lagartas/tubo. Foram avaliados a duração e a viabilidade das fases larval e pupal e o peso de pupas machos e fêmeas.

Todos os experimentos seguiram o delineamento inteiramente casualizado. Os dados foram submetidos à análise de variância (teste $F$ ), sendo as médias comparadas pelo teste de Tukey $(P \leq 0,05)$.

\section{RESULTADOS E DISCUSSÃO}

No primeiro experimento, com folhas, não houve diferença na duração da fase larval de $P$. operculella em função do genótipo de batata utilizado, mas em todos estes tratamentos, este período foi mais curto que 0 obtido em folhas de tomate. As viabilidades larval e pupal e a duração da fase pupal não foram afetadas pelos diversos tratamentos (TABELA 1). Embora os genótipos de batata também não tenham afetado o peso de pupas, houve uma tendência de redução no valor desse parâmetro quando as lagartas foram criadas em folhas de tomate (TABELA 2). Assim, as pupas machos provenientes de folhas de tomate apresentaram peso menor do que as obtidas nos demais tratamentos, com exceção do genótipo NYL 235-4; já em relação às pupas fêmeas, o peso em tomate foi inferior ao apresentado por insetos provenientes dos genótipos Apuã e Aracy.

Com base nesses resultados, verifica-se que os genótipos NYL 235-4 e N 140-201, apesar de apresentarem tricomas glandulares nas folhas, não exerceram efeito adverso sobre o desenvolvimento de $P$. operculella. É possível que as lagartas dessa espécie sejam pouco afetadas pelos tricomas devido à rápida penetração nas folhas, permanecendo, conseqüentemente, pouco tempo em contato com os mesmos. Posteriormente, alimentam-se somente do mesofilo foliar, ficando protegidas dos exsudatos tóxicos dos tricomas.

Musmeci et al. (1997) também não constataram efeito negativo de duas introduções de $S$. berthaultii e do híbrido K 507.46, ambos com tricomas A e B, sobre 
TABELA 1 - Médias ( \pm EP) de duração e viabilidade das fases larval e pupal de Phthorimaea operculella alimentada com folhas de diferentes genótipos de batata e da cv. Santa Clara de tomate. Temp.: $25 \pm 2^{\circ} \mathrm{C}$; UR: $60 \pm 10 \%$ e fotofase: $12 \mathrm{~h}$.

\begin{tabular}{|c|c|c|c|c|}
\hline \multirow[t]{2}{*}{ Genótipos } & \multicolumn{2}{|c|}{ Fase larval } & \multicolumn{2}{|c|}{ Fase pupal } \\
\hline & Duração ${ }^{1}$ & Viabilidade $^{1}$ & Duração ${ }^{1}$ & Viabilidade $^{1}$ \\
\hline & dias & $\%$ & dias & $\%$ \\
\hline Tomate & $16,36 \pm 0,24 a$ & $71,67 \pm 5,00 a$ & $8,48 \pm 0,13 a$ & $80,83 \pm 4,98 a$ \\
\hline Itararé & $15,08 \pm 0,15 b$ & $80,00 \pm 4,84 a$ & $8,60 \pm 0,20 \quad a$ & $85,00 \pm 3,57 a$ \\
\hline Apuã & $14,65 \pm 0,13 b$ & $85,00 \pm 6,31 a$ & $8,10 \pm 0,23 a$ & $93,33 \pm 3,47 a$ \\
\hline NYL 235-4 & $14,63 \pm 0,07 b$ & $86,67 \pm 3,33 a$ & $8,07 \pm 0,21 a$ & $85,83 \pm 4,13 a$ \\
\hline N 140-201 & $14,49 \pm 0,12 b$ & $73,33 \pm 5,67 a$ & $8,28 \pm 0,20 a$ & $91,67 \pm 5,12 a$ \\
\hline Aracy & $14,36 \pm 0,12 b$ & $68,33 \pm 5,24 a$ & $8,18 \pm 0,16 a$ & $82,46 \pm 6,31$ \\
\hline
\end{tabular}

${ }^{1}$ Médias seguidas da mesma letra não diferem pelo teste de Tukey $(P \leq 0,05)$.

TABELA 2 - Médias ( \pm EP) do peso de pupas de Phthorimaea operculella provenientes de lagartas alimentadas em folhas de diferentes genótipos de batata e da cv. Santa Clara de tomate. Temp.: $25 \pm 2^{\circ} \mathrm{C}$; UR: $60 \pm 10 \%$ e fotofase: $12 \mathrm{~h}$.

\begin{tabular}{lcc}
\hline \multicolumn{1}{c}{ Genótipos } & \multicolumn{1}{c}{ Machos } & Fêmeas \\
\hline N 140-201 & $9,12 \pm 0,31^{1} \mathrm{a}$ & $10,09 \pm 0,32 \mathrm{ab}$ \\
Itararé & $8,98 \pm 0,22 \mathrm{a}$ & $10,21 \pm 0,35 \mathrm{ab}$ \\
Aracy & $8,81 \pm 0,29 \mathrm{a}$ & $10,59 \pm 0,17 \mathrm{a}$ \\
Apuã & $8,51 \pm 0,14 \mathrm{a}$ & $10,29 \pm 0,18 \mathrm{a}$ \\
NYL 235-4 & $8,05 \pm 0,31 \mathrm{ab}$ & $9,68 \pm 0,18 \mathrm{ab}$ \\
Tomate & $7,33 \pm 0,32 \mathrm{a}$ & $9,23 \pm 0,22 \mathrm{~b}$ \\
\hline
\end{tabular}

${ }^{1}$ Médias seguidas da mesma letra, nas colunas, não diferem pelo teste de Tukey $(P \leq 0,05)$.

o estabelecimento de lagartas recém-eclodidas de $P$. operculella; além disso, verificaram valores relativamente altos de viabilidade larval para insetos criados em $S$. berthaultii. Resultados similares foram obtidos por Westedt et al. (1998) que observaram elevada sobrevivência larval (96\%), após $48 \mathrm{~h}$ da infestação em folhas do genótipo NYL 235-4. Entretanto, Musmeci et al. (1997) verificaram redução no peso pupal e na fecundidade da traça em clones de $S$. berthaultii e híbridos de $S$. tuberosum $\times$ S. berthaultii.

As folhas de tomate (comparadas às de batata), foram menos favoráveis ao inseto, provocando alongamento do período larval e redução no peso pupal. Doreste \& Nieves (1968) também consideraram folhas de tomate como alimento menos satisfatório para $P$. operculella do que folhas de batata e fumo visto que, em tomate, ocorreu menor viabilidade da fase imatura e menor longevidade de adultos. Gomaa et al. (1978) obtiveram menor peso de pupas (machos e fêmeas) em folhas de tomate que em folhas e tubérculos de batata.

No segundo experimento, houve alongamento da fase larval para insetos criados nos clones NYL 235-4 e N 140-201 em relação aos criados nos demais tratamentos. O menor período larval foi observado quando os insetos foram criados em folhas de 'Apuã', diferindo de todos os outros tratamentos (TABELA 3).

Verificou-se que os tubérculos dos clones NYL 235-4 e N 140-201 proporcionaram os menores valores de viabilidade larval, sendo que em NYL 235-4 a viabilidade (20\%) foi inferior a todos os tratamentos e em $\mathrm{N} \mathrm{140-201(46 \% )}$ foi semelhante apenas à observada na cultivar Aracy (68\%). Nos demais tratamentos, foram constatados valores superiores a $80 \%$ (TABELA 3).

Em relação aos parâmetros viabilidade e duração da fase pupal (TABELA 3) e peso de pupas fêmeas (TABELA 4), não foram observadas diferenças significativas entre os tratamentos. Entretanto, as pupas machos provenientes do genótipo NYL 235-4 apresentaram peso menor que as encontradas no genótipo Itararé (TABELA 4).

De modo geral, verificou-se que a viabilidade e a duração da fase larval foram os parâmetros mais afetados pelos diversos tratamentos. Este resultado é importante, considerando-se que é nesta fase que o inseto causa danos à cultura.

Neste experimento, verificou-se que os tubérculos dos clones NYL 235-4 e N 140-201 provocaram redução na viabilidade larval e alongamento desta fase, sendo estes efeitos mais pronunciados em NYL 235-4. Estes resultados evidenciam que os tubérculos destes clones apresentam algum fator, possivelmente químico e ou mecânico, que afeta adversamente o desenvolvimento do inseto. Entretanto, outros estudos devem ser realizados para 0 conhecimento dos mecanismos de resistência envolvidos.

Fatores de resistência a $P$. operculella têm sido constatados em tubérculos de várias espécies selvagens como Solanum sucrense, $S$. pinnatisectum, $S$. sparsipilum e $S$. phureja, entre outras, sendo transferidos com sucesso para germoplasmas cultivados (Ortiz et al., 1990; Chavez et al., 1988).

Em relação a $S$. berthaultii e aos clones resultantes de cruzamentos envolvendo esta espécie, a avaliação da resistência tem ficado restrita aos efeitos 
TABELA 3 - Médias ( \pm EP) de duração e viabilidade das fases larval e pupal de Phthorimaea operculella alimentada com tubérculos de diferentes genótipos de batata e folhas de 'Apuã'. Temp.: $25 \pm 2^{\circ} \mathrm{C}$; UR: $60 \pm 10 \%$ e fotofase: $12 \mathrm{~h}$.

\begin{tabular}{|c|c|c|c|c|}
\hline \multirow{2}{*}{ Genótipos } & \multicolumn{2}{|c|}{ Fase larval } & \multicolumn{2}{|c|}{ Fase pupal } \\
\hline & Duração $^{1}$ & Viabilidade $^{1}$ & Duração $^{1}$ & Viabilidade $^{1}$ \\
\hline & dias & $\%$ & dias & $\%$ \\
\hline NYL 235-4 & $26,00 \pm 0,32 a$ & $20,00 \pm 3,54 d$ & $7,97 \pm 0,29 a$ & $96,00 \pm 4,00$ \\
\hline N 140-201 & $21,55 \pm 0,49 b$ & $46,00 \pm 6,40 \mathrm{c}$ & $7,83 \pm 0,14 a$ & $100,00 \pm 0,00$ \\
\hline Aracy & $18,15 \pm 0,40 \mathrm{c}$ & $68,00 \pm 4,36 \mathrm{bc}$ & $8,02 \pm 0,16 a$ & $95,13 \pm 3,30$ \\
\hline Itararé & $17,82 \pm 0,29 c$ & $82,00 \pm 6,82 a b$ & $8,00 \pm 0,11 \mathrm{a}$ & $91,29 \pm 2,73$ \\
\hline Apuã (Tubérc.) & $16,61 \pm 0,16 c$ & $88,00 \pm 5,15 a b$ & $7,56 \pm 0,08 a$ & $96,53 \pm 2,34$ \\
\hline Apuã (Folhas) & $14,31 \pm 0,24 d$ & $91,00 \pm 1,87 a$ & $7,71 \pm 0,15 a$ & $91,00 \pm 2,91$ \\
\hline
\end{tabular}

${ }^{1}$ Médias seguidas da mesma letra não diferem pelo teste de Tukey $(P \leq 0,05)$.

TABELA 4 - Médias ( \pm EP) do peso de pupas de Phthorimaea operculella provenientes de lagartas alimentadas em tubérculos de diferentes genótipos de batata e em folhas de 'Apuã'. Temp.: $25 \pm 2^{\circ} \mathrm{C}$; UR: $60 \pm 10 \%$ e fotofase: $12 \mathrm{~h}$.

\begin{tabular}{|c|c|c|}
\hline \multirow{2}{*}{ Genótipos } & Machos & Fêmeas \\
\hline & ------------------ & | ---------------- \\
\hline Itararé & $8,68 \pm 0,21^{1} a$ & $9,43 \pm 0,44 a$ \\
\hline N 140-201 & $8,60 \pm 0,38 a b$ & $9,43 \pm 0,66 a$ \\
\hline Apuã (Tubérculos) & $8,47 \pm 0,07 a b$ & $9,50 \pm 0,26 a$ \\
\hline Aracy & $8,43 \pm 0,21 a b$ & $9,56 \pm 0,71 \mathrm{a}$ \\
\hline Apuã (Folhas) & $7,72 \pm 0,22 a b$ & $8,74 \pm 0,25 a$ \\
\hline NYL 235-4 & $7,45 \pm 0,41 b$ & $8,19 \pm 0,37 a$ \\
\hline
\end{tabular}

${ }^{1}$ Médias seguidas da mesma letra, nas colunas, não diferem pelo teste de Tukey $(P \leq 0,05)$.

dos tricomas glandulares presentes nas folhas, sendo dada pouca atenção à avaliação da resistência em tubérculos. O bom nível de resistência encontrado nos tubérculos de NYL 235-4, nesta pesquisa, indica o grande potencial deste genótipo como fonte de resistência a $P$. operculella.

\section{AGRADECIMENTOS}

Ao Centro Nacional de Pesquisa de Hortaliças/ EMBRAPA e The McKnight Foundation, pelo suporte financeiro e fornecimento de genótipos e ao Instituto Agronômico de Campinas, pelo fornecimento das cultivares de batata.

\section{REFERÊNCIAS BIBLIOGRÁFICAS}

AHMED, S.; HUQ, S.B.; TALUKDER, F.A. Susceptibility of potato to potato moth in field and storage. Bangladesh Journal Agricultural Science, v.18, p.289-290, 1991.

CHAVEZ, R.; SCHMIEDICHE, P.E.; JACKSON, M.T.; RAMAN, K.V. The breeding potential of wild potato species resistant to the potato tuber moth, Phthorimaea operculella (Zeller). Euphytica, v.39, p.123-132, 1988.
DORESTE, E.; NIEVES, M. Estudios de laboratorio sobre el ciclo biologico del minador de la hoja del tabaco, papa y tomate, Gnorimoschema operculella Zeller. Agronomía Tropical, v.18, p.461-474, 1968.

FENEMORE, P.G. Susceptibility of potato cultivars to potato tuber moth, Phthorimaea operculella Zell. (Lepidoptera: Gelechiidae). New Zealand Journal of Agricultural Research, v.23, p.539-546, 1980.

FRANÇA, F.H.; TINGEY, W.M. Solanum berthaultii Hawkes affects the digestive system, fat body and ovaries of the Colorado potato beetle. American Potato Journal, v.71, p.405-410, 1994.

GOMAA, A.A.; EL-SHERIF, S.; HEMEIDA, I.A. On the biology of the potato tuber moth, Phthorimaea operculella Zeller (Lepidoptera: Gelechiidae): I. Effect of larval diet. Journal of Applied Entomology, v.86, p.290-294, 1978.

GURR, G.M.; SYMINGTON, C.A. Resistance to the potato tuber moth, Phthorimaea operculella (Zeller) (Lepidoptera: Gelechiidae), in potato (Solanum tuberosum L.) tubers. Australian Journal of Entomology, v.37, p.49-51, 1998.

GYAWALI, B.K. Ovipositional preference of tuber moth Phthorimaea (Gnorimoschema) operculella Zeller on five varieties of potatoes in Nepal. Tropical Pest Management, v.35, p.106-107, 1989.

MUSMECI, S.; CICCOLI, R.; DI GIOIA, V.; SONNINO, A.; ARNONE, S. Leaf effects of wild species of Solanum and interspecific hybrids on growth and behavior of the potato tuber moth, Phthorimaea operculella Zeller. Potato Research, v.40, p.417-430, 1997.

OJERO, M.F.; MUEKE, J.M. Resistance of four potato varieties to the potato tuber moth Phthorimaea operculella (Zell.) in storage. Insect Science and its Application, v.6, p.205-207, 1985.

ORTIZ, R.; IWANAGA, M.; RAMAN, K.V.; PALACIOS, M. Breeding for resistance to potato tuber moth, Phthorimaea operculella (Zeller), in diploid potatoes. Euphytica, v.50, p.119-125, 1990.

PALACIOS, M.; RAMAN, K.V.;ALCÁZAR, J. Control integrado de la polilla de la papa Phthorimaea opercullela (Zeller). In: TALLER DE MANEJO INTEGRADO DE PLAGAS, Balcarce, 1993. Memorias. Balcarce: INTA, 1993. p.63-71.

PLAISTED, R.L.; TINGEY, W.M.; STEFFENS, J.C. The germplasm release of NYL 235-4, a clone with resistance to the Colorado potato beetle. American Potato Journal, v.69, p.843-847, 1992. 
PRATISSOLI, D.; BARBOSA, S.M.M.V.; USSIT, M.T.; PARRA, J.R.P. Aprimoramento da técnica de criação de Phthorimaea operculella (Zeller, 1873) visando ao seu controle biológico: determinação do número ideal de lagartas por tubérculos de batata para máxima produção de insetos. In: REUNIÃO PAULISTA DE INICIAÇÃO CIENTÍFICA EM CIÊNCIAS AGRÁRIAS, 4., Piracicaba, 1993. Resumos. Piracicaba: FEALQ, 1993. p.43.

RADCLIFFE, E.B. Insects pests of potato. Annual Review of Entomology, v.27, p.173-204, 1982.

RAMAN, K.V.; PALACIOS, M. Screening potato for resistance to potato tuberworm. Journal of Economic Entomology, v.75, p.47-49, 1982.

STEIN, C.P. Resistência de cultivares de Solanum tuberosume de híbridos de $S$. tuberosum x S. berthaultii a Myzus persicae (Sulzer), Liriomyza huidobrensis (Blanchard), Phthorimaea operculella (Zeller) e pragas de solo. Piracicaba, 1998. 74p. Tese (Doutorado) - Escola Superior de Agricultura "Luiz de Queiroz", Universidade de São Paulo.
TINGEY, W.M.; GIBSON, R.W. Feeding and mobility of the potato leafhopper impaired by glandular trichomes of Solanum berthaultii and $S$. polyadenium. Journal of Economic Entomology, v.71, p.856-858, 1978.

TINGEY, W.M.; SINDEN, S.L. Glandular pubescence, glycoalkaloid composition and resistance to the green peach aphid, potato leafhopper and potato fleabeetle in Solanum berthaultii. American Potato Journal, v.59, p.95-106, 1982.

VARELA, L.G.; BERNAYS, E.A. Behavior of newly hatched potato tuber moth larvae, Phthorimaea operculella Zell. (Lepidoptera: Gelechiidae), relation to their host plants. Journal of Insect Behavior, v.1, p.261-275, 1988.

WESTEDT, A.L.; DOUCHES, D.S.; PETT, W.; GRAFIUS, E.J. Evaluation of natural and engineered resistance mechanisms in Solanum tuberosum for resistance to Phthorimaea operculella (Lepidoptera, Gelechiidae). Journal of Economic Entomology, v.91, p.552-556, 1998.

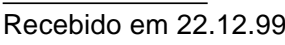

